\title{
Cerebral oxidative metabolism mapping in four genetic mouse models of anxiety and mood disorders
}

Denis Matrova,b, Tanel Kaart ${ }^{c}$, Laurence Lanfumey ${ }^{d}$, Rafael Maldonado ${ }^{e}$, Trevor Sharp ${ }^{f}$, Rosa M. Torderag ${ }^{g}$ Paul A. Kelly ${ }^{h}$, Bill Deakin ${ }^{i} \&$ Jaanus Harro ${ }^{a}{ }^{*}$

${ }^{a}$ Division of Neuropsychopharmacology, Department of Psychology, University of Tartu, Ravila 14A Chemicum, 50411 Tartu, Estonia

${ }^{\mathrm{b}}$ Department of Neuroscience, Graduate School of Medicine, Kyoto University, Yoshida-konoe-cho, Sakyo-ku, Kyoto, 606-8501, Japan

c Institute of Veterinary Medicine and Animal Sciences, Estonian University of Life Sciences, Kreutzwaldi 1, 51014 Tartu, Estonia

${ }^{d}$ Centre de Psychiatrie et Neuroscience, INSERM U 894, 2 ter rue d'Alésia, 75014 Paris, France e Laboratory of Neuropharmacology, Department of Experimental and Health Sciences, Universitat Pompeu Fabra, Barcelona, Spain

${ }^{f}$ University Department of Pharmacology, Mansfield Road, Oxford, OX1 3QT, United Kingdom. g Department of Pharmacology and Toxicology, University of Navarra, Pamplona, Spain

${ }^{\mathrm{h}}$ Centres for Cognitive and Neural Systems, University of Edinburgh, Scotland, United Kingdom 'Neuroscience and Psychiatry Unit, Division of Neuroscience and Experimental Psychology, School of Biological Sciences, Faculty of Biology, Medicine and Health, University of Manchester, United Kingdom

${ }^{*}$ Corresponding author. Tel.: +372 7376657; fax: +372 7375900. E-mail address:

jaanus.harro@ut.ee (J. Harro)

\section{Abstract}

The psychopathology of depression is highly complex and the outcome of studies on animal models is divergent. In order to find brain regions that could be metabolically distinctively active across a variety of mouse depression models and to compare the interconnectivity of brain regions of wild-type and such genetically modified mice, histochemical mapping of oxidative metabolism 
was performed by the measurement of cytochrome oxidase activity. We included mice with the heterozygous knockout of the vesicular glutamate transporter $\left(\mathrm{VGLUT}_{1}^{-/+}\right)$, full knockout of the cannabinoid 1 receptor $\left(\mathrm{CB}^{-/-}\right)$, an anti-sense knockdown of the glucocorticoid receptor (GRi) and overexpression of the human 5-hydroxytryptamine transporter (h5-HTT). Altogether 76 mouse brains were studied to measure oxidative metabolism in one hundred brain regions, and the obtained dataset was submitted to a variety of machine learning algorithms and multidimensional scaling. Overall, the top brain regions having the largest contribution to classification into depression model were the lateroanterior hypothalamic nucleus, the anterior part of the basomedial amygdaloid nucleus, claustrum, the suprachiasmatic nucleus, the ventromedial hypothalamic nucleus, and the anterior hypothalamic area. In terms of the patterns of interregional relationship between wild-type and genetically modified mice there was little overall difference, while the most deviating brain regions were cortical amygdala and ventrolateral and ventral posteromedial thalamic nuclei. The GRi mice that most clearly differed from their controls exhibited deviation of connectivity for a number of brain regions, such as ventrolateral thalamic nucleus, the intermediate part of the lateral septal nucleus, the anteriodorsal part of the medial amygdaloid nucleus, the medial division of the central amygdaloid nucleus, ventral pallidum, nucleus of the vertical limb of the diagonal band, anteroventral parts of the thalamic nucleus and parts of the bed nucleus of the stria terminalis. Conclusively, the GRi mouse model was characterized by changes in the functional connectivity of the extended amygdala and stress response circuits.

Keywords: Depression; Animal model; Genetically modified mice; Oxidative metabolism; Cytochrome oxidase histochemistry

\section{Introduction}

The psychopathology of depression is highly complex (Drysdale et al., 2017; Duman et al., 2016) and likely to involve components common to all depressed patients as well as individually distinct pathogenetic roads (Harro and Oreland, 2001). Animal models of depression have significantly contributed to drug development since the emergence of the original antidepressants, but while 
the understanding of the neurobiology of depression has also advanced, the increasing diversity of models has brought about a large variety of neurobiological findings that are not well shared (Cryan and Holmes, 2005; Harro, 2013). Much of this variance may be the result of model-specific alterations and not of general interest, but a real conflict exists between the pursuit of reductionistic animal models and the recognition that the diagnosis of depression represents huge heterogeneity of clinical conditions. Hence it is not surprising if diagnostic criteria of mental illness do not translate easily into animal models of a single gene mutation; however, smaller building blocks or "intermediate phenotypes" of a psychiatric syndrome should be modeled successfully (Donaldson and Hen, 2015). A major attempt to detect common genome-wide differences by the NewMood consortium (Deakin et al., 2011) using a panel of animal models of depression that were to share the depression endophenotypes - anhedonia, anxiety, and helplessness highlighted many candidate genes (Hoyle et al., 2011; Aso et al., 2011; Tordera et al., 2011; Fabre et al., 2011; Lisowski et al., 2011; Kanarik et al., 2011), but essentially did not produce a consistent, cross-model picture. This suggests that at the gene expression level the model-specific aspects prevail. In order to study the coherence in the neurobiologies of depression at a higher level of brain organization, regional oxidative metabolism mapping by cytochrome oxidase histochemistry was performed in a number of rat models of depression. Cytochrome c oxidase (COX; EC 1.9.3.1) is a rate-limiting enzyme in the mitochondrial electron transport chain, where its activity mirrors the generation of ATP. Cytochrome $\mathrm{c}$ oxidase activity is relatively stable in time and hence serves as a good indicator for linking neurometabolic and behavioural profiles (Wong-Riley et al., 1998). While individual rat models again yielded only a few similarities in regional oxidative metabolism (Harro et al., 2011), possibly owing to specifics of models as well as limited statistical power, the analysis across four rat models of depression suggested common patterns of vulnerability and stress response (Harro et al., 2014). Hence we have carried out a joint analysis of oxidative metabolism as measured in four of the core genetic mouse models of the NewMood project (Deakin et al., 2011). The mouse depression models included the heterozygous knockout of the vesicular glutamate transporter (VGLUT ${ }^{-/+}$) mice (Garcia-Garcia et al., 2009), full knockout of the cannabinoid 1 receptor $\left(\mathrm{CB}^{-/-}\right)$mice (Martin et al., 2002), and an anti-sense knockdown of the glucocorticoid receptor (GRi) mice (Lanfumey et al., 2000). These models were developed on guidance of current understanding of the neurobiology of affective disorders, and in all four models the genetically modified mice had some pertinent differences with the respective wild-type (WT) animals in conventional neuropsychopharmacology tests. The VGLUT1 ${ }^{-/+}$mice exhibit increased anxiety in the light-dark compartment test and elevated immobility in the forced swimming test (Tordera et al., 
2007) as well as increased vulnerability to anhedonia after chronic stress (García-García et al., 2009) that was reversed by antidepressant treatment (García-García et al., 2009; Muñoz-Cobo et al., 2017). The $\mathrm{CB}^{-/-}$mice exhibit anxiety-like behaviour in the light/dark compartment test (Martin et al., 2002) and under bright illumination in the elevated plus-maze (Haller et al., 2004), increased immobility in the tail suspension test (Aso et al., 2008), as well as increased anhedonia after chronic unpredictable mild stress (Martin et al., 2002). The depressive-like phenotype of $\mathrm{CB}^{-/}$mice was significantly attenuated by the systemic administration of imipramine or fluoxetine (Mato et al., 2007) and completely reversed by the local administration of BDNF in the hippocampus (Aso et al., 2008). GRi mice exhibited marked alterations in depressive-like and anxiety-like behaviors, together with a significant increase in fear-related behaviors. They also showed decreased cell proliferation and altered levels of neuroplastic and epigenetic markers in the hippocampus (Paizanis et al., 2010, Boulle et al., 2016). We also used mice overexpressing the human 5-hydroxytryptamine transporter (h5-HTT) (Line et al., 2011) as these animals have higher clearance of 5-HT and, theoretically, lower serotonin function. These mice have differences in fear learning that depend on experimental conditions (Barkus et al., 2014; McHugh et al., 2015).

Hence, this study had the following aims: i) to find brain regions that could be metabolically distinctively active in a variety of mouse depression models, and ii) to compare the interconnectivity of brain regions of wild-type and genetically modified mice.

\section{Experimental procedures}

\subsection{Animals}

$\mathrm{CB}^{-/-}$mice were of CD1 genetic background (Ledent et al., 1999). $\mathrm{VGLUT}_{1}^{-/+}$mice were of C57BL/6N genetic background (Wojcik et al., 2004) and bred from heterozygous fathers and WT mothers (Harlan, France). Glucocorticoid receptor-impaired (GRi) mice were of F1 B6/C3H genetic background (Bengel et al., 1998). h5-HTT overexpressing mice were of CBA x C57BL/6J hybrid background (Jennings et al., 2006). The number of available animals was as follows: GRi 28 (14 genetically modified, GM, and $14 \mathrm{WT}$ ), CB1 ${ }^{-/-} 9$ (4 GM and 5 WT mice), VGLUT ${ }^{-/+} 21$ (10 GM and 11 WT), and h5-HTT 18 (9 GM and 9 WT). All mice in the current study were male, experimentally naive and were not stressed deliberately. Experimental procedures and animal husbandry were conducted according to the principles of laboratory animal care as detailed in the European Communities Council Directive (2003/65/EC). 


\subsection{Cytochrome oxidase histochemistry and image analysis}

Frozen brains of all mice were shipped on dry ice to the University of Tartu where all histochemical experiments were conducted as previously described (Mällo et al., 2009). Frozen brains were sectioned on cryostat into $50 \mu \mathrm{m}$ thick coronal sections. Sections from mice of each genetic model with the corresponding WTs were processed histochemically together in a balanced manner. Optical density scores of cytochrome oxidase activity were standardized and converted into Z scores within each genetic model to exclude the effects of uncontrolled variation in lab conditions, as brains from different genetic models were processed separately. Therefore only the relative differences between GM and WT mice can be compared across animal models. Five values, each from a different brain region, were not successfully obtained, and multiple imputation by chained equations (MICE) R package (Buuren and Groothuis-Oudsdoorn, 2011) was employed using a "predictive mean matching" approach to fill in the missing values.

\subsection{Data analysis}

All statistical analyses were performed using R software version 3.4.

In conditions where the total number of observations is less than the number of predictor variables while predictors are additionally collinear, machine learning offers a number of advantages (Thomaz et al., 2006) but there is variety of techniques that are not simple to select a priori. Hence, different machine learning $(\mathrm{ML})$ algorithms were used to find the most important brain regions distinguishing GM and WT mice. Repeated cross-validation was applied to achieve conservative estimates of prediction accuracies. At first all algorithms were applied on full dataset incorporating all animal models. As oxidative metabolism raw data from different animal models can be fairly different (Harro et al., 2014), additional more granular analyses were performed, applying similar analysis to reduced datasets. The following analyses were performed: 1) each animal model was removed from the dataset in turn, 2) data of animals from a single model were analyzed in turn, 3) because the vulnerability to anxiety of h5-HTT genotype is uncertain (see Discussion), it was recoded into separate category of "resistant" and the analysis was performed on full dataset to distinguish "resistant", "control" and "vulnerable" mice. Also, because the data for brain regions were highly collinear, the parameter estimates may be unstable and it is hard to estimate the contribution of each variable to the final model; therefore, the full dataset was 
analyzed once again, with only half of the variables representing regional COX activity were sampled on each iteration of the algorithm's evaluation. Additionally, to study the effect of data selection, the relative importance of brain regions on full dataset was estimated based on different subsets of mice (10\% of samples with average prediction accuracy, and $10 \%$ of samples with the highest or with the lowest prediction accuracies). In general, maximum uncertainty linear discriminant analysis (MLDA; Thomaz et al., 2006) algorithm was the most successful on larger datasets. In more granular analyses, penalyzed discriminant analysis (PDA; Hastie et al., 1995) and linear discriminant analysis (LDA; Venables and Ripley, 2003) performed slightly better. All other ML approaches, such as tree-based algorithms, neural nets, and naive Bayes classifiers, were slightly worse in their accuracy, so the easier to interpret linear discriminant models were preferred for presentation of results. Initial evaluation of $M L$ algorithms was done using the caret $\mathrm{R}$ package (Kuhn and Johnson, 2013), MLDA was performed with HiDimDA R package (Pedro Duarte Silva, 2011) and PDA was performed with penalizedLDA R package (Witten and Tibshirani, 2011). More detailed description of performed ML analyses is presented in Supplementary material. The receiver operating characteristic (ROC) analysis (Fawcett, 2006) was used to test the differences between and to assess the prediction accuracy of single brain regions distinguishing WT and GM mice. The ROC curves depict sensitivity (probability of correctly classified GM mice) and specificity (probability of correctly classified WT mice) for all possible values of the brain region's activity and visualize in such a way simultaneously two aspects of the prediction accuracy. Results of ROC analysis can be summarized as a single metric by computing the area under the ROC curve $(A \cup C)$ and by testing its difference from 0.5 (AUC=0.5 corresponds to random appointment of mice into groups irrespective of regional oxidative metabolism). The ROC curves were plotted with plotROC R package and the statistical significance of AUC was calculated by performing 10,000 permutation tests with the sigr R package. Since the effects of 100 brain regions were tested independently, the more conservative threshold for statistically significant effects was set at $p<0.005$.

To assess overall inter-regional connectivity patterns, the procedures as described in our earlier study in the rat (Harro et al., 2014) were used. The correlation matrices were calculated for both WT and GM mice including all brain regions and the multidimensional scaling (MDS) algorithm was applied on the difference of the two correlation matrixes. The MDS discovers the most important patterns of regional metabolic activity distinguishing GM and WT mice and allows projecting the results into low-dimensional space. The correlation matrixes were plotted with corrplot $\mathrm{R}$ package 
and the MDS was performed with ade4 R package. The analyses were performed with dataset including all animals from all models and additionally with the GRi group showing the strongest dissimilarities between GM and WT mice.

\section{Results}

\subsection{Machine learning}

When the full dataset was analyzed, the accuracy of MLDA predictions of whether a mouse belonged to the depression models or wild-type was $0.62 \pm 0.09$ (with the theoretical maximum being 1.0). The results for classification accuracy of the best algorithm for each dataset are presented in Table 1. Overall, the accuracy of classification was modest when the dataset was composed of mice from several genotypes. Model-wise, the GRi mice had the largest proportion of correct classifications $(0.77 \pm 0.15)$. This also implied that analyzing the data without GRi mice the overall prediction accuracy decreased while omitting any other genotype almost did not change the overall prediction accuracy. Reduction of predictor variables (that is, brain regions) to the random sample of 50 had little effect on the prediction accuracy.

Overall, the top four brain regions having the largest contribution to classification into depression model were the lateroanterior hypothalamic nucleus (LA), the anterior part of the basomedial amygdaloid nucleus (BMA), claustrum (CL), and the suprachiasmatic nucleus (SCh). By single animal models, $\mathrm{CL}$ and $\mathrm{SCh}$ occurred in top five of discriminating regions for more than one model: $\mathrm{CL}$ was the most discriminative brain region for $\mathrm{GRi}$ mice and second most salient for $\mathrm{CB}_{1}$ mice, $\mathrm{SCh}$ was the most significant for VGLUT1 model and fourth more important for GRi mice. Estimating the relative importance of brain regions on full dataset based on different subsets of mice (10 percentage of samples with average prediction accuracy, and 10 percentage of samples with the highest or with the lowest prediction accuracies) did not change the results, as the top six brain regions remained the same (with LA at very the top) and overall the ranks of brain regions were remarkably similar. Recoding h5-HTT mice into its own phenotype ("resistant") deteriorated the overall accuracy of machine learning (MLDA accuracy score: $0.56 \pm 0.09$ ), suggesting that even this phenotypically distinct genetic modification contributed to the overall "depressed" configuration of oxidative activity. 
The results of bootstrap analysis to estimate the magnitude and stability of discriminant vector coefficients in MLDA for whole dataset and separately for the GRi model in the 10,000 times bootstrapping are presented in Figure 1, and the results of bootstrap analysis in both MLDA and PDA for all animal models separately are presented in Supplementary Figure 1. Altogether the results of twelve brain regions with the largest discriminant vector coefficients are presented. The coefficients of the discriminant vector were the most stable for GRi animal model, the stability on the full dataset was somewhat worse. Other three animal models had poor coefficient stability and there were little agreement in variable coefficients between MLDA and PDA algorithms suggesting the absence of general patterns in regional oxidative metabolism distinguishing GM and WT mice.

\subsection{Receiver Operating Characteristic analysis}

The results of ROC analysis essentially affirmed the results of machine learning algorithms. The ROC curves of the top six brain regions with the lowest $p$-values as assessed in the full dataset (LA, $\mathrm{BMA}, \mathrm{CL}, \mathrm{SCh}$, the ventromedial hypothalamic nucleus, VMH, and the anterior hypothalamic area, posterior part, AHP) are presented on Figure 2 for the whole dataset and for each animal model separately. On the full dataset LA, BMA and SCh were significantly discriminative $(p<0.005)$. However, the classification accuracy into WT or GM was poor (all AUC < 0.7). Distinguishing GM and WT mice in case of GRi animal model, there were 16 brain regions with $p<0.005$ (CL, LA, AHP, $\mathrm{BMA}, \mathrm{SCh}, \mathrm{VMH}$, anterior hypothalamic area, anterior part, AHA, ectorhinal cortex, Ect, zona incerta, ZI, substantia innominata, SI, basolateral amygdaloid nucleus, anterior part, BLA, paraventricular thalamic nucleus, PV, medial amygdaloid nucleus, anteriodorsal, MeAD, lateral entorhinal cortex, Lent, dorsal endopiriform nucleus, Den, and lateral hypothamic area, LH). Thirteen of these brain regions allowed to classify the mice into "control" and "vulnerable" groups with good accuracy ( $A \cup C \geq 0.8$ ). As to other mouse models separately, none of brain regions had statistically significant effect at the $p<0.005$ level.

\subsection{Correlation analysis and multidimensional scaling}

Brain regional COX histochemistry data were, overall, highly inter-correlated. In the whole dataset the median correlation for control, wild-type mice was 0.82 (inter quartile range $I Q R=0.10$ ), whilst it was lower at $0.74(I Q R=0.11)$ for GM mice (Supplementary Figures 2 and 3). In case of GRi mice, the inter-regional correlations were lower and more variable but the difference between GM and 
WT was larger: the median correlation score for GRi mice was $0.59(I Q R=0.24)$ and for the corresponding WT mice $0.78(I Q R=0.15)$.

In the whole dataset of four models there was little difference in the patterns of inter-regional relationship between "control" and "vulnerable" mice. This is indicated by most of the brain regions being located in the centre of the multidimensional scaling figure (Figure $3 \mathrm{~A}$ ) and limited deviation of the rest, the largest changes in functional connectivity from the other brain regions being observed for the ventrolateral thalamic nucleus, cortical amygdala, and ventral posteromedial thalamic nucleus (as derived from the average deviation of first five dimensions in the MDS). In case of the GRi model the differences in regional connectivity patterns were larger, and thirty-two brain regions were differentially connected to the rest to a larger extent than the VL in the overall connectivity model (Figure 3B and Supplementary Figure 4). Notably deviating regions from the wild-type functional connectivity included, besides the $\mathrm{V} L$, the intermediate part of the lateral septal nucleus (LSI), the anteriodorsal part of the medial amygdaloid nucleus (MeAD) and the medial division of the central amygdaloid nucleus (CeM), ventral pallidum (VP), nucleus of the vertical limb of the diagonal band (VDB), anteroventral parts of the thalamic nucleus and parts of the bed nucleus of the stria terminalis.

Multidimensional scaling was used as a hypothesis-free test of connectivity but also to enhance the statistical power of the analysis. As the brain regions deviating most of the rest of the brain in the GRi mice are known as involved in stress response, we carried out an exploratory correlation analysis of the brain regions of the extended amygdala and central stress response circuits (Figure 4). Particularly large reduction in the GRi mice as compared to wild-type was observed in correlations of oxidative metabolism between several nuclei of the amygdala and between these amygdaloid nuclei and and some regions of the hypothalamus and the cingulate cortex.

\section{Discussion}

We had previously analyzed jointly the oxidative metabolism mapping data from four principally different rat models of affective disorders, and while each model separately yielded somewhat specific findings, the overall analysis permitted conclusions on the patterns of regional oxidative metabolism pertinent to vulnerability to stress, the stress effects and the diathesis-stress model (Harro et al., 2014). Herewith we report common aspects derived from four genetically modified mouse models of depression. The models represent the hyperactivity of the hypothalamo- 
pituitary-adrenal (HPA) system, as well as deficits in serotonergic, glutamatergic, and cannabinoid signaling.

It should first be noted that inter-regional correlation was higher in the mouse brain as compared to the rat brain. Whether this applies over a larger selection of strains and genetic backgrounds remains to be confirmed. Within the constraints of the four selected models, irrespective of differences in their genetic background, WT mice of all models were highly homogeneous in their neural metabolism. The correlation matrix of control mice also had a higher median correlation as compared to the genetically modified counterparts, and the machine learning algorithms in general also had more success in correctly classifying the WT animals. The genetically modified mouse brain was, overall, functionally less strongly coupled, as revealed by lower overall correlation. This resembles the lower overall inter-regional correlation as observed in the rat brain after chronic stress (Harro et al., 2014). The mice in the present study were not intentionally stressed and the potential impact of stress remains to be investigated; it is however tempting to speculate that genetically modified mice, at least those considered as models of depression, could be more sensitive to animal house conditions than their wild-type counterparts. Such an interpretation is indirectly supported by the largest deviations observed in the GRi model that specifically targets the stress axis regulation.

Region-wise, all applied methods of analysis suggested a few brain areas that across all models had higher oxidative metabolism than the wild-type, while there were large differences between the models. The latter may well relate to differences in genetic background, but it should also be noted that phenotype-wise, the four models are far from similar. The VGLUT1 ${ }^{-/+}$mice have high anxiety and elevated immobility in the forced swimming test (Tordera et al., 2007) as well as increased vulnerability to anhedonia (García-García et al., 2009) that was reversed by antidepressant treatment (García-García et al., 2009; Muñoz-Cobo et al., 2017). The CB1/-/- mice also exhibit anxiety-like behaviour (Martin et al., 2002; Haller et al., 2004) and increased anhedonia after chronic unpredictable mild stress (Martin et al., 2002). The GRi mice, however, demonstrate lower immobility in the forced swim test, whereas the time spent in the open arms of EPM was elevated (Barden et al., 2005; Boulle et al., 2016). The fourth model, mice overexpressing the human 5hydroxytryptamine transporter (h5-HTT) was considered a vulnerability model owing to reduced synaptic serotonin but there is hardly any direct evidence for depression-like phenotype; instead, the h5-HTT mice spent more time on the open arms and had shorter latency to enter them in EPM (Jennings et al., 2006), and their open field and marble burying profiles were similar to WT controls 
(Jennings et al., 2006; Line et al., 2011). Fear learning was found to be impaired in the 5-HTT overexpression mice, and hemodynamic responses in amygdala in response to aversive cues was lower (Barkus et al., 2014). Fear response appears as reduced in the 5-HTT overexpressing mice in the conditions where the aversive cues do not suggest the occurrence of adverse events with certainty (McHugh et al., 2015). Curiously, h5-HTT mice also weigh less and have smaller bodies, whereas the amount of food they consume does not differ from WT mice (Jennings et al., 2006; Pringle et al., 2008). Altogether, the behavioural similarities and differences between the mouse models appear to have no simple correspondence with similarities or differences in regional oxidative metabolism, but this is not different from the previous findings with rat models (Harro et al., 2011). All mouse models in the present analyses had been included in the "NewMood" project - a cooperative EU research framework to identify new molecules involved in triggering mood disorders (Deakin et al., 2011). Previously, gene expression in frontal cortex, hippocampus and raphe has been jointly analyzed in VGLUT1, $\mathrm{CB}_{1}$, and GRi mouse models as well as the serotonin transporter knockout mice (Hoyle et al., 2011). Genes belonging to gene ontology categories related to metabolic processes and energy demand were differentially expressed in the genetically modified mice, but there was little similarity in the gene expression magnitudes or direction between the models (Hoyle et al., 2011). The conclusion from gene expression profiling in rat models was rather similar (Alttoa et al., 2010; Kanarik et al., 2011; van den Hove et al., 2013). It has been said that animal genetic models of mood disorders and anxiety require "sanity checks" at intermediate steps on the ladder of biological causality that try to fill the vast space separating a malfunctioning neurotransmitter pathway from a behavioural phenotype resembling a psychiatric symptom (Wong and Josselyn, 2016). While in the rat the level of regional oxidative metabolism was informative on common neurobiology behind separate models (Harro et al., 2014), the results of a similar analysis in mouse models were less coherent. Nevertheless, a few clues to similarities between mouse models surfaced. While using the whole dataset, the most salient brain regions to define the "depressed" mouse included the claustrum, anterior and suprachiasmatic hypothalamic regions and basomedial amygdala. The BMA neurons encode anxiety-associated contextual features and BMA activation decreases fear-related freezing and high-anxiety states (Adhikari et al., 2015). Anterior hypothalamus, of which LA and AHP are parts, is activated during discrete proximal threats, such as predatory attacks (Hakvoort Schwerdtfeger and Menard, 2008). The implication of SCh in anxiety and fear may by involved by contribution to the cognitive processing of fearful stimuli (Chaudhury and Colwell, 2002) but also a more general role in biological rhythms that are impaired in depression (McCarthy and Welsh, 2012). That claustrum was one of the brain 
regions highlighted in this cross-model analysis is highly interesting: Electrical stimulation of claustrum in dogs can elicit both active and passive defensive behaviours (Vakolyuk et al., 1983) and this brain region, while not commonly considered in depression research, has been the subject of interest in understanding how information flow between regions of cerebral cortex is coordinated (Crick and Koch, 2005). In the current study, claustrum was a particularly important variable in CB1 and GRi mouse models. Previously it has been found to be hypoactive in human patients suffering from depression (Dunn et al., 2002; Fitzgerald et al., 2008), and in socially defeated rats (Kanarik et al., 2011). Brain-derived neurotropic factor (BDNF) may serve as an important mediator between stress-driven activation of HPA axis and subsequent post-synaptic remodelling that may lead to mood disorders (Massart et al., 2012). For instance, after 7 days of periodic immobilization stress in mice, BDNF mRNA expression was strongly reduced in claustrum and elevated in lateral hypothalamus and medial amygdala (Smith et al., 1995). Differences in stress-induced BDNF mRNA expression and transcriptional control have been found in hippocampus of GRi and $\mathrm{CB}_{1}$ animal models (Alboni et al., 2011; Aso et al., 2008).

While all mouse models contributed to overall model, their relative share was affected by both the apparent size of effect of the genotype on oxidative metabolism as well as by the number of animals available. Hence detailed discussion of findings in each model separately would remain premature, however, except for the GRi model for which the classification into control vs. model groups was most successful. Glucocorticoid receptor-impaired mice were created to investigate the role of HPA axis dysfunction in mood disorders (Boulle et al., 2016). The activity of HPA axis depends on the hypothalamic secretion of corticotrophin releasing hormone (CRH) and arginine vasopressin (AVP). Corticosterone exerts a negative feedback control on CRH and AVP secretion by binding to low- affinity glucocorticoid receptors and high-affinity mineralocorticoid receptors (Massart et al., 2012). Glucocorticoid receptor-impaired mice are resistant to suppression of corticosterone secretion by an exogenous steroid dexamethasone (Barden et al., 1997).

Dexamethasone non-suppression can be considered the "intermediate phenotype" of human depression of melancholic type (Juruena et al., 2017). In addition to the HPA axis disturbances, hyper-responsiveness under stress of the serotonergic system has been noted (Froger et al., 2004; Linthorst et al., 2000).

Our machine learning results on GRi mice fit well into the narrative of hyperactive HPA, as well as the reactivity of serotonergic system in GRi mice. In the top 5 five most important variables for machine learning, three regions were from hypothalamus: LA, AHP, \& SCh. In addition, claustrum 
and the anterior part of the basolateral amygdala $(B L A)$ featured prominently in the machine learning results. In GRi animal model, the genetically modified animals had a higher than WT mice mean metabolic activity in all of these brain regions. Previously, increased metabolic activity was recorded in the paraventricular nucleus of hypothalamus in the rat, but not in the other five hypothalamic nuclei studied (including LA and SCh) in the congenitally helpless rat model (Shumake et al., 2001). Differences in the COX activity levels in hypothalamus and median raphe were also previously recorded in the chronically stressed rats: brain metabolism in MnR was elevated after chronical nonsocial stress and lowered in LA after chronic social stress (Harro et al., 2011, 2014).

Multidimensional scaling offers the advantages of hypothesis free testing of the changes in regional correlation patterns within the brain. In order to specify the connectivity changes at the level of known networks we focused on the extended amygdala and stress response circuits in the GRi mice, given that several brain regions belonging to this network featured as outliers in the MDS. A robust anatomical connection exists between the central amygdaloid nucleus and the BNST (Ch'ng et al., 2018), and in the GRi mice the oxidative metabolism between divisions of these brain areas was uncoupled. Functional connectivity of medial and basolateral nuclei of amygdala with regions of hypothalamus and the BNST were also weakened. Amongst these prominent alterations, the reduction of activity coupling between medial amygdaloid nucleus and the medial preoptic nucleus is of interest given that in the rat, deviation of oxidative metabolism in the latter brain region were most strongly associated with deviations in trait-wise social behaviour (Kanarik and Harro, 2018). While no major decline was observed in the GRi mice with regard to the association of the level of oxidative metabolism between amygdala and cingulate, a significant connection in depression (Pezawas et al., 2005), a major drop in activity coupling was observed between a cingulate area and the BNST that mediates the amygdaloid outputs.

In conclusion, regional oxidative metabolism as revealed by cytochrome oxidase histochemistry was found to be highly inter-correlated in the mouse brain, and joint analysis of four genetic mouse models of affective disorders revealed large differences between the models but also highlighted several brain regions implicated in their shared characteristics. Involvement of a few hypothalamic nuclei, basomedial amygdala and claustrum was common. The GRi mouse model was characterized by several alterations in oxidative metabolism and changes in the functional 
connectivity of the extended amygdala and stress response circuits. Further studies examining additional mouse models should test the generalizability of these findings.

\section{Conflict of interest}

The authors have no conflict of interest to declare.

\section{Acknowledgements}

This research has been supported by the EC 6FP Integrated Project NEWMOOD, the Hope for Depression Research Foundation and the Institute for the Study of Affective Neuroscience, and the Estonian Ministry of Education and Science project IUT20-40.

\section{References}

Adhikari, A., Lerner, T.N., Finkelstein, J., Pak, S., Jennings, J.H., Davidson, T.J., Ferenczi, E., Gunaydin, L.A., Mirzabekov, J.J., Ye, L., Kim, S.-Y., Lei, A., Deisseroth, K., 2015. Basomedial amygdala mediates top-down control of anxiety and fear. Nature 527, 179-185. https://doi.org/10.1038/nature15698

Alboni, S., Tascedda, F., Corsini, D., Benatti, C., Caggia, F., Capone, G., Barden, N., Blom, J.M.C., Brunello, N., 2011. Stress induces altered CRE/CREB pathway activity and BDNF expression in the hippocampus of glucocorticoid receptor-impaired mice. Neuropharmacology 60, 1337-1346. https://doi.org/10.1016/j.neuropharm.2011.01.050

Alttoa, A., Kõiv, K., Hinsley, T.A., Brass, A., Harro, J., 2010. Differential gene expression in a rat model of depression based on persistent differences in exploratory activity. Eur. Neuropsychopharmacol. 20, 288-300. https://doi.org/10.1016/j.euroneuro.2009.09.005

Aso, E., Ozaita, A., Serra, M.A., Maldonado, R., 2001. Genes differentially expressed in $\mathrm{CB}_{1}$ knockout mice: Involvement in the depressive-like phenotype. Eur. Neuropsychopharmacol. 21, 11-22. https://doi.org/10.1016/i.euroneuro.2010.06.007

Aso, E., Ozaita, A., Valdizán, E.M., Ledent, C., Pazos, A., Maldonado, R., Valverde, O., 2008. BDNF impairment in the hippocampus is related to enhanced despair behavior in CB1 knockout mice. J. Neurochem. 105, 565-572. https://doi.org/10.1111/j.1471-4159.2007.05149.x 
Barden, N., Shink, E., Labbé, M., Vacher, R., Rochford, J., Mocaër, E., 2005. Antidepressant action of agomelatine (S 20098) in a transgenic mouse model. Prog. Neuropsychopharmacol. Biol. Psychiatry 29, 908-916. https://doi.org/10.1016/j.pnpbp.2005.04.032

Barden, N., Stec, I.S., Montkowski, A., Holsboer, F., Reul, J.M., 1997. Endocrine profile and neuroendocrine challenge tests in transgenic mice expressing antisense RNA against the glucocorticoid receptor. Neuroendocrinology 66, 212-220. https://doi.org/10.1159/000127240

Barkus, C., Line, S.J., Huber, A., Capitao, L., Lima, J., Jennings, K., Lowry, J., Sharp, T., Bannerman, D.M., McHugh, S.B., 2014. Variation in serotonin transporter expression modulates fearevoked hemodynamic responses and theta-frequency neuronal oscillations in the amygdala. Biol. Psychiatry 75, 901-908. https://doi.org/10.1016/j.biopsych.2013.09.003

Bengel, D., Murphy, D.L., Andrews, A.M., Wichems, C.H., Feltner, D., Heils, A., Mössner, R., Westphal, H., Lesch, K.P., 1998. Altered brain serotonin homeostasis and locomotor insensitivity to 3, 4-methylenedioxymethamphetamine ("Ecstasy") in serotonin transporterdeficient mice. Mol. Pharmacol. 53, 649-655. https://doi.org/10.1124/mol.53.4.649

Boulle, F., Velthuis, H., Koedam, K., Steinbusch, H.W., van den Hove, D.L.A., Kenis, G., Gabriel, C., Mocaer, E., Franc, B., Rognan, D., Mongeau, R., Lanfumey, L., 2016. Behavioral and neurochemical characterization of TrkB-dependent mechanisms of agomelatine in glucocorticoid receptor-impaired mice. Eur. Neuropsychopharmacol. 26, 65-77. https://doi.org/10.1016/j.euroneuro.2015.11.003

Buuren, S., Groothuis-Oudshoorn, K., 2011. mice: Multivariate imputation by chained equations in R. J. Stat. Softw. 45. http://dx.doi.org/10.18637/jss.v045.i03

Ch'ng, S., Fu, J., Brown, R.M., McDougall, S.J., Lawrence, A.J., 2018. The intersection of stress and reward:BNST modulation of aversive and appetitive states. Prog. Neuro-Psychopharmacol. Biol Psychiat., in press; https://doi.org/10.1016/j.pnpbp.2018.01.005.

Chaudhury, D., Colwell, C.S., 2002. Circadian modulation of learning and memory in fearconditioned mice. Behav. Brain Res. 133, 95-108. https://doi.org/10.1016/S01664328(01)00471-5

Crick, F.C., Koch, C., 2005. What is the function of the claustrum? Philos. Trans. R. Soc. Lond. B. Biol. Sci. 360, 1271-1279. https://doi.org/10.1098/rstb.2005.1661

Cryan, J.F., Holmes, A., 2005. The ascent of mouse: Advances in modelling human depression and anxiety. Nat. Rev. Drug Discov. 4, 775-90. https://doi.org/10.1038/nrd1825 
Deakin, J.F., Harro, J., Anderson, I.M., 2011. NewMood: A productive European model of collaboration for translational research in depression. Eur. Neuropsychopharmacol. 21, 1-2. htps://doi.org/10.1016/j.euroneuro.2010.11.008

Donaldson, Z.R., Hen, R., 2015. From psychiatric disorders to animal models: A bidirectional and dimensional approach. Biol. Psychiatry 77, 15-21. https://doi.org/10.1016/j.biopsych.2014.02.004

Drysdale, A.T., Grosenick, L., Downar, J., Dunlop, K., Mansouri, F., Meng, Y., Fetcho, R.N., Zebley, B., Oathes, D.J., Etkin, A., Schatzberg, A.F., Sudheimer, K., Keller, J., Mayberg, H.S., Gunning, F.M., Alexopoulos, G.S., Fox, M.D., Pascual-Leone, A., Voss, H.U., Casey, B.J., Dubin, M.J., Liston, C., 2017. Resting-state connectivity biomarkers define neurophysiological subtypes of depression. Nat.Med. 23, 28-38. https://doi.org/10.1038/nm.4246

Duman, R.S., Aghajanian, G.K., Sanacora, G., Krystal, J.H., 2016. Synaptic plasticity and depression: New insights from stress and rapid-acting antidepressants. Nat. Med. 22, 238-249. htps://doi.org/10.1038/nm.4050

Dunn, R.T., Kimbrell, T.A., Ketter, T.A., Frye, M.A., Willis, M.W., Luckenbaugh, D.A., Post, R.M., 2002. Principal components of the Beck Depression Inventory and regional cerebral metabolism in unipolar and bipolar depression. Biol. Psychiatry 51, 387-399. https://doi.org/10.1016/S0006-3223(01)01244-6

Fabre, V., Massart, R., Rachalski, A., Jennings, K., Brass, A., Sharp, T., Lesch, K.P., Lanfumey, L., Hamon, M., 2011. Differential gene expression in mutant mice overexpressing or deficient in the serotonin transporter: A focus on urocortin 1. Eur. Neuropsychopharmacol. 21, 33-44. https://doi.org/10.1016/j.euroneuro.2010.10.004

Fawcett, T., 2006. An introduction to ROC analysis. Pattern Recognit. Lett. 27, 861-874. https://doi.org/10.1016/j.patrec.2005.10.010

Fitzgerald, P.B., Laird, A.R., Maller, J., Daskalakis, Z.J., 2008. A meta-analytic study of changes in brain activation in depression. Hum. Brain Mapp. 29, 683-695. https://doi.org/10.1002/hbm.20426

Froger, N., Palazzo, E., Boni, C., Hanoun, N., Saurini, F., Joubert, C., Dutriez-Casteloot, I., Enache, M., Maccari, S., Barden, N., Cohen-Salmon, C., Hamon, M., Lanfumey, L., 2004. Neurochemical and behavioral alterations in glucocorticoid receptor-impaired transgenic mice after chronic mild stress. J. Neurosci. 24, 2787-2796. https://doi.org/10.1523/JNEUROSCI.4132-03.2004 Garcia-Garcia, A.L., Elizalde, N., Matrov, D., Harro, J., Wojcik, S.M., Venzala, E., Ramírez, M.J., Del Rio, J., Tordera, R.M., 2009. Increased vulnerability to depressive-like behavior of mice with 
decreased expression of VGLUT1. Biol. Psychiatry 66, 275-282.

https://doi.org/10.1016/j.biopsych.2009.02.027

Hakvoort Schwerdtfeger, R.M., Menard, J.L., 2008. The lateral hypothalamus and anterior hypothalamic nucleus differentially contribute to rats' defensive responses in the elevated plus-maze and shock-probe burying tests. Physiol. Behav. 93, 697-705.

https://doi.org/10.1016/j.physbeh.2007.11.011

Haller, J., Varga, B., Ledent, C., Barna, I., Freund, T.F., 2004. Context-dependent effects of CB1 cannabinoid gene disruption on anxiety-like and social behaviour in mice. Eur. J. Neurosci. 19, 1906-1912. https://doi.org/10.1111/j.1460-9568.2004.03293.x

Harro, J., 2013. Animal models of depression vulnerability. Curr. Top. Behav. Neurosci. 14, 29-54. https://doi.org/10.1007/7854_2012_221.

Harro, J., Kanarik, M., Matrov, D., Panksepp, J., 2011. Mapping patterns of depression-related brain regions with cytochrome oxidase histochemistry: Relevance of animal affective systems to human disorders, with a focus on resilience to adverse events. Neurosci. Biobehav. Rev. 35, 1876-1889. https://doi.org/10.1016/j.neubiorev.2011.02.016

Harro, J., Kanarik, M., Kaart, T., Matrov, D., Kõiv, K., Mällo, T., Del Río, J., Tordera, R.M., Ramirez, M.J., 2014. Revealing the cerebral regions and networks mediating vulnerability to depression: oxidative metabolism mapping of rat brain. Behav. Brain Res., 267, 83-94. https://doi.org/10.1016/j.bbr.2014.03.019

Harro, J., Oreland, L., 2001. Depression as a spreading adjustment disorder of monoaminergic neurons: A case for primary implication of the locus coeruleus. Brain Res. Rev. 38, 79128. https://doi.org/10.1016/S0165-0173(01)00082-0

Hastie, T., Buja, A., Tibshirani, R., 1995. Penalized Discriminant Analysis. Ann. Stat. 23, 73-102. https://doi.org/10.1214/aos/1176324456

Hoyle, D., Juhasz, G., Aso, E., Chase, D., del Rio, J., Fabre, V., Hamon, M., Lanfumey, L., Lesch, K.-P., Maldonado, R., Serra, M.-A., Sharp, T., Tordera, R., Toro, C., Deakin, J.F.W., 2011. Shared changes in gene expression in frontal cortex of four genetically modified mouse models of depression. Eur. Neuropsychopharmacol. 21, 3-10. https://doi.org/10.1016/j.euroneuro.2010.09.011

Jennings, K.A., Loder, M.K., Sheward, W.J., Pei, Q., Deacon, R.M.J., Benson, M.A., Olverman, H.J., Hastie, N.D., Harmar, A.J., Shen, S., Sharp, T., 2006. Increased expression of the 5-HT transporter confers a low-anxiety phenotype linked to decreased 5-HT transmission. J. Neurosci. 26, 8955-8964. https://doi.org/10.1523/JNEUROSCI.5356-05.2006 
Juruena, M.F., Bocharova, M., Agustini, B., Young, A.H., 2017. Atypical depression and non-atypical depression: Is HPA axis function a biomarker? A systematic review. J. Affect. Disord. https://doi.org/10.1016/j.jad.2017.09.052

Kanarik, M., Harro, J., 2018. Sociability trait and regional cerebral oxidative metabolism in rats: Predominantly nonlinear relations. Behav. Brain Res. 337, 186-192.

Kanarik, M., Alttoa, A., Matrov, D., Kõiv, K., Sharp, T., Panksepp, J., Harro, J., 2011. Brain responses to chronic social defeat stress: Effects on regional oxidative metabolism as a function of a hedonic trait, and gene expression in susceptible and resilient rats. Eur. Neuropsychopharmacol. 21, 92-107. https://doi.org/10.1016/j.euroneuro.2010.06.015 Kuhn, M., Johnson, K., 2013. Applied Predictive Modeling. Springer, New York Lanfumey, L., Mannoury La Cour, C., Froger, N., Hamon, M., 2000. 5-HT-HPA interactions in two models of transgenic mice relevant to major depression. Neurochem. Res. 25, 1199-1206. https://doi.org/10.1023/A:100768381

Ledent, C., Valverde, O., Cossu, G., Petitet, F., Aubert, J.F., Beslot, F., Böhme, G.A., Imperato, A., Pedrazzini, T., Roques, B.P., Vassart, G., Fratta, W., Parmentier, M., 1999. Unresponsiveness to cannabinoids and reduced addictive effects of opiates in CB1 receptor knockout mice. Science 283, 401-404. https://doi.org/10.1126/science.283.5400.401

Line, S.J., Barkus, C., Coyle, C., Jennings, K.A., Deacon, R.M., Lesch, K.P., Sharp, T., Bannerman, D.M., 2011. Opposing alterations in anxiety and species-typical behaviours in serotonin transporter overexpressor and knockout mice. Eur. Neuropsychopharmacol. 21, 108-116. https://doi.org/10.1016/j.euroneuro.2010.08.005

Linthorst, A.C., Flachskamm, C., Barden, N., Holsboer, F., Reul, J.M., 2000. Glucocorticoid receptor impairment alters CNS responses to a psychological stressor: An in vivo microdialysis study in transgenic mice. Eur. J. Neurosci. 12, 283-291.

Lisowski, P., Juszczak, G.R., Goscik, J., Wieczorek, M., Zwierzchowski, L., Swiergiel, A.H., 2011. Effect of chronic mild stress on hippocampal transcriptome in mice selected for high and low stress-induced analgesia and displaying different emotional behaviors. Eur. Neuropsychopharmacol. 21, 45-62. https://doi.org/10.1016/j.euroneuro.2010.08.004 Mällo, T., Matrov, D., Kõiv, K., Harro, J., 2009. Effect of chronic stress on behavior and cerebral oxidative metabolism in rats with high or low positive affect. Neuroscience 164, 963-974. https://doi.org/10.1016/j.neuroscience.2009.08.041 
Martin, M., Ledent, C., Parmentier, M., Maldonado, R., Valverde, O., 2002. Involvement of CB1 cannabinoid receptors in emotional behaviour. Psychopharmacology (Berl.) 159, 379-387. https://doi.org/10.1007/s00213-001-0946-5

Massart, R., Mongeau, R., Lanfumey, L., 2012. Beyond the monoaminergic hypothesis:

Neuroplasticity and epigenetic changes in a transgenic mouse model of depression. Philos. Trans. R. Soc. Lond. B. Biol. Sci. 367, 2485-2494. https://doi.org/10.1098/rstb.2012.0212

Mato, S., Aso, E., Castro, E., Martin, M., Valverde, O., Maldonado, R., Pazos, A., 2007. CB 1 knockout mice display impaired functionality of 5- $\mathrm{HT}_{1 \mathrm{~A}}$ and 5- $\mathrm{HT}_{2 \mathrm{~A} / \mathrm{c}}$ receptors. J. Neurochem. 1003, 2111-2120. https://doi.org/10.1111/j.1471-4159.2007.04961.x

McCarthy, M.J., Welsh, D.K., 2012. Cellular circadian clocks in mood disorders. J. Biol. Rhythms 27, 339-352. https://doi.org/10.1177/0748730412456367

McHugh, S.B., Barkus, C., Lima, J., Glover, L.R., Sharp, T., Bannerman, D.M., 2015. SERT and uncertainty: Serotonin transporter expression influences information processing biases for ambiguous aversive cues in mice. Genes Brain Behav. 14, 330-336. https://doi.org/10.1111/gbb.12215

Muñoz-Cobo, I., Belloch, F.B., Díaz-Perdigón, T., Puerta, E., Tordera, R.M., 2017. SIRT2 inhibition reverses anhedonia in the VGLUT1+/- depression model. Behav. Brain Res. 335, 128-131. https://doi.org/10.1016/j.bbr.2017.07.045

Païzanis, E., Renoir, T., Lelievre, V., Saurini, F., Melfort, M., Gabriel, C., Barden, N., Mocaër, E., Hamon, M., Lanfumey, L., 2017. Behavioural and neuroplastic effects of the new-generation antidepressant agomelatine compared to fluoxetine in glucocorticoid receptor-impaired mice. Int J Neuropsychopharmacol. 13,759-74. doi: 10.1017/S1461145709990514.

Pedro Duarte Silva, A., 2011. Two-group classification with high-dimensional correlated data: A factor model approach. Comput. Stat. Data Anal. 55, 2975-2990. https://doi.org/10.1016/j.csda.2011.05.002

Pezawas, L., Meyer-Lindenberg, A., Drabant, E.M., Verchinski B.A., Munoz, K.E., Kolachana, B.S., Egan, M.F., Mattay, V.S., Hariri, A.R., Weinberger, D.R., 2005. 5-HTTLPR polymorphism impacts human cingulate-amygdala interactions: A genetic susceptibility mechanism for depression. Nature Neurosci. 8, 828-834.

Pringle, A., Jennings, K.A., Line, S., Bannerman, D.M., Higgs, S., Sharp, T., 2008. Mice overexpressing the 5-hydroxytryptamine transporter show no alterations in feeding 
behaviour and increased non-feeding responses to fenfluramine. Psychopharmacology (Berl.) 200, 291-300. https://doi.org/10.1007/s00213-008-1206-8

Shumake, J., Edwards, E., Gonzalez-Lima, F., 2001. Hypermetabolism of paraventricular hypothalamus in the congenitally helpless rat. Neurosci. Lett. 311, 45-48. https://doi.org/10.1016/S0304-3940(01)02142-5

Smith, M.A., Makino, S., Kim, S.Y., Kvetnansky, R., 1995. Stress increases brain-derived neurotropic factor messenger ribonucleic acid in the hypothalamus and pituitary. Endocrinology 136, 3743-3750. https://doi.org/10.1210/endo.136.9.7649080

Thomaz, C.E., Kitani, E.C., Gillies, D.F., 2006. A maximum uncertainty LDA-based approach for limited sample size problems - with application to face recognition. J. Braz. Comput. Soc. 12, 7-18. https://doi.org/10.1007/BF03192391

Tordera, R.M., Garcia-García, A.L., Elizalde, N., Segura, V., Aso, E., Venzala, E., Ramírez, M.J., Del Rio, J., 2011. Chronic stress and impaired glutamate function elicit a depressive-like phenotype and common changes in gene expression in the mouse frontal cortex. Eur. Neuropsychopharmacol. 21, 23-32. https://doi.org/10.1016/j.euroneuro.2010.06.016

Tordera, R.M., Totterdell, S., Wojcik, S.M., Brose, N., Elizalde, N., Lasheras, B., Del Rio, J., 2007. Enhanced anxiety, depressive-like behaviour and impaired recognition memory in mice with reduced expression of the vesicular glutamate transporter 1 (VGLUT1). Eur. J. Neurosci. 25, 281-290. https://doi.org/10.1111/j.1460-9568.2006.05259.x

Vakolyuk, N.I., Kosterina, A.V., Shlumukova, A.R., 1983. Electrical stimulation of the claustrum and conditioned-reflex activity. Neurosci. Behav. Physiol. 13, 158-163. https://doi.org/10.1007/BF01148844

Van den Hove, D.L., Kenis, G., Brass, A., Opstelten, R., Rutten, B.P., Bruschettini, M., Blanco, C.E., Lesch, K.P., Steinbusch, H.W., Prickaerts, J., 2013. Vulnerability versus resilience to prenatal stress in male and female rats; implications from gene expression profiles in the hippocampus and frontal cortex. Eur Neuropsychopharmacol. 23, 1226-46. https://doi.org/10.1016/j.euroneuro.2012.09.011

Venables, W.N., Ripley, B.D., 2003. Modern Applied Statistics with S, 4th ed. Springer, New York Witten, D.M., Tibshirani, R., 2011. Penalized classification using Fisher's linear discriminant. J. R. Stat. Soc. Ser. B Stat. Methodol. 73, 753-772. https://doi.org/10.1111/j.14679868.2011.00783.x

Wojcik, S.M., Rhee, J.S., Herzog, E., Sigler, A., Jahn, R., Takamori, S., Brose, N., Rosenmund, C., 2004. An essential role for vesicular glutamate transporter 1 (VGLUT1) in postnatal 
development and control of quantal size. Proc. Natl. Acad. Sci. U. S. A. 101, 7158-7163. https://doi.org/10.1073/pnas.0401764101

Wong, A.H., Josselyn, S.A., 2016. Caution when diagnosing your mouse with schizophrenia: The use and misuse of model animals for understanding psychiatric disorders. Biol. Psychiatry 79, 32-8. https://doi.org/10.1016/j.biopsych.2015.04.023

Wong-Riley, M.T.T., Nie, F., Hevner, R.F., Liu, S., 1998. Brain cytochrome oxidase: Functional significance and bigenomic regulation in the CNS. In: Gonzales-Lima F, editor. Cytochrome oxidase in neuronal metabolism and Alzheimer's disease. Plenum Press, New York, pp 1-53. 
Table 1. The overall prediction accuracies, and the sensitivities and specificities $( \pm \mathrm{SE})$ by animal models achieved with machine learning algorithms applied on different datasets.

\begin{tabular}{|c|c|c|c|c|c|c|c|c|c|c|}
\hline \multirow[t]{2}{*}{ Dataset } & \multirow{2}{*}{$\begin{array}{l}\text { Best } \\
\text { algorithm* }\end{array}$} & \multirow{2}{*}{$\begin{array}{c}\text { Overall } \\
\text { accuracy }\end{array}$} & \multicolumn{2}{|c|}{ h5-HTT } & \multicolumn{2}{|c|}{ CB1 } & \multicolumn{2}{|c|}{ VGLUT1 } & \multicolumn{2}{|c|}{ GRi } \\
\hline & & & Sensitivity & Specificity ${ }^{\#}$ & Sensitivity & Specificity & Sensitivity & Specificity & Sensitivity & Specificity \\
\hline Full dataset & MLDA & $0.62 \pm 0.09$ & $0.51 \pm 0.32$ & $0.66 \pm 0.32$ & $0.28 \pm 0.45$ & $0.53 \pm 0.50$ & $0.51 \pm 0.33$ & $0.68 \pm 0.27$ & $0.69 \pm 0.23$ & $0.73 \pm 0.23$ \\
\hline Dataset with 50 predictors & MLDA & $0.62 \pm 0.09$ & $0.51 \pm 0.32$ & $0.65 \pm 0.31$ & $0.22 \pm 0.41$ & $0.48 \pm 0.50$ & $0.53 \pm 0.31$ & $0.67 \pm 0.28$ & $0.71 \pm 0.23$ & $0.73 \pm 0.23$ \\
\hline Without h5-HTT mice & MLDA & $0.64 \pm 0.12$ & NA & NA & $0.35 \pm 0.48$ & $0.56 \pm 0.50$ & $0.47 \pm 0.32$ & $0.64 \pm 0.29$ & $0.73 \pm 0.22$ & $0.76 \pm 0.23$ \\
\hline Without CB1 mice & MLDA & $0.67 \pm 0.10$ & $0.57 \pm 0.32$ & $0.64 \pm 0.32$ & NA & NA & $0.52 \pm 0.32$ & $0.64 \pm 0.30$ & $0.75 \pm 0.20$ & $0.77 \pm 0.22$ \\
\hline Without VGLUT1 mice & MLDA & $0.61 \pm 0.11$ & $0.54 \pm 0.31$ & $0.60 \pm 0.33$ & $0.26 \pm 0.44$ & $0.52 \pm 0.50$ & NA & NA & $0.70 \pm 0.23$ & $0.71 \pm 0.24$ \\
\hline Without GRi mice & PDA & $0.58 \pm 0.13$ & $0.56 \pm 0.31$ & $0.72 \pm 0.29$ & $0.78 \pm 0.42$ & $0.46 \pm 0.50$ & $0.44 \pm 0.31$ & $0.57 \pm 0.28$ & NA & NA \\
\hline Only h5-HTT mice & PDA & $0.52 \pm 0.22$ & $0.43 \pm 0.32$ & $0.61 \pm 0.33$ & NA & NA & NA & NA & NA & NA \\
\hline Only CB1 mice & PDA & $0.60 \pm 0.36$ & NA & NA & $0.67 \pm 0.47$ & $0.52 \pm 0.50$ & NA & NA & NA & NA \\
\hline Only VGLUT1 mice & LDA & $0.59 \pm 0.21$ & NA & NA & NA & NA & $0.48 \pm 0.34$ & $0.68 \pm 0.29$ & NA & NA \\
\hline Only GRi mice & LDA & $0.77 \pm 0.15$ & NA & NA & NA & NA & NA & NA & $0.77 \pm 0.22$ & $0.77 \pm 0.23$ \\
\hline
\end{tabular}

${ }^{*}$ MLDA - Maximum uncertainty Linear Discriminant Analysis, PDA - Penalised Discriminant Analysis, LDA - Linear Discriminant Analysis.

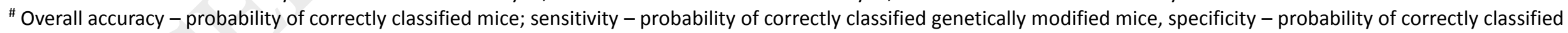
wild type mice.

NA - not applicable. 


\section{Legends to Figures}

Figure 1. Variable importance coefficients from maximum uncertainty linear discriminant analysis in full dataset (left) and in the GRi animal model (right). The mean of each coefficient is plotted in red. Individual coefficient values from bootstrap sampling are plotted in gray. Just a fraction of individual values is presented to prevent overplotting. The percentage numbers on the right depict the proportion of bootstrap trials where discriminant coefficients were different from zero for each variable. Blue dashed and solid lines represent distance from zero equal to two and three standard deviations, respectively, computed from all scores. Abbreviations as in Figure 3.

Figure 2. ROC curves for six most significant brain regions distinguishing GM and WT mice in full dataset (black lines) plus ROC curves of the same brain regions for each animal model separately (color lines). The area under the curve (AUC) values and significance levels of the full dataset are presented besides each graph. Abbreviations as in Figure 3.

Figure. 3. Results of multidimensional scaling comparing the regional energy metabolism correlation patterns of "control" and "vulnerable" mice based on all and only GRi data. Interrelations of differences in correlations are fitted in 2-dimensional space represented by the axes of the figure. The distance between any two brain regions, each represented by its abbreviation, is a relative indicator of their functional inter-relatedness in terms of association strength. Abbreviations: AcbC accumbens nucleus core, AcbSH accumbens nucleus shell,Al agranular insular cortex, AAD anterior amygdaloid area dorsal part, Aco anterior cortical amygdaloid nucleus, AHA anterior hypothalamic area anterior part, AHP anterior hypothalamic area posterior part, $A O D$ anterior olfactory nucleus dorsal part, $\mathrm{AOL}$ anterior olfactory nucleus lateral part, AOM anterior olfactory nucleus medial part, AOV anterior olfactory nucleus ventral part, ADP anterodorsal preoptic nucleus, AD anterodorsal thalamic nucleus, Am anteromedial thalamic nucleus, AVDM anteroventral thalamic nucleus dorsomedial part, AVVL anteroventral thalamic nucleus ventrolateral part, BLA basolateral amygdaloid nucleus anterior part, BMA basomedial amygdaloid nucleus anterior part, BSTLD bed nucleus of the stria terminalis lateral division dorsal part, BSTLV bed nucleus of the stria terminalis lateral division ventral part, BSTM bed nucleus of the stria terminalis medial division, Cpu caudate putamen (striatum), CeM central amygdaloid nucleus medial division, $\mathrm{CM}$ central medial thalamic nucleus, $\mathrm{Cg} 1$ cingulate cortex area $1, \mathrm{Cg} 2$ cingulate cortex area $2, \mathrm{Cg} / \mathrm{RS}$ cingulate/retrosplenial cortex, CL claustrum, DG dentate gyrus, Den dorsal endopiriform nucleus, DR dorsal raphe nucleus, DLO dorsolateral orbital cortex, DM dorsomedial hypothalamic nucleus, DMPAG dorsomedial periaqueductal gray, Ect ectorhinal 
cortex, CA1 field CA1 of hippocampus, CA2 field CA2 of hippocampus, CA3 field CA3 of hippocampus, FrA frontal association cortex, GP globus pallidus, IL infralimbic cortex, IPR interpeduncular nucleus rostral subnucleus, Lent lateral entorhinal cortex, LHb lateral habenular nucleus, LH lateral hypothalamic area, LO lateral orbital cortex, LPAG lateral periaqueductal gray, LPO lateral preoptic area, LSI lateral septal nucleus intermediate part, LA lateroanterior hypothalamic nucleus, LC locus coeruleus, MeAD medial amygdaloid nucleus anterior dorsal, MHb medial habenular nucleus, MM medial mammillary nucleus medial part, MO medial orbital cortex, MPA medial preoptic area, MPOM medial preoptic nucleus medial part, MS medial septal nucleus, MnPO median preoptic nucleus, MnR median raphe nucleus, MDL mediodorsal thalamic nucleus lateral part, MDM mediodorsal thalamic nucleus medial part, HDB nucleus of the horizontal limb of the diagonal band, VDB nucleus of the vertical limb of the diagonal band, PT paratenial thalamic nucleus, PV paraventricular thalamic nucleus, PVA paraventricular thalamic nucleus anterior part, PAG periaqueductal gray, Pir piriform cortex, Pn pontine nuclei, $\mathrm{PH}$ posterior hypothalamic area, PrL prelimbic cortex, PrS presubiculum, M1 primary motor cortex, S1BF primary somatosensory cortex barrel field, V1 primary visual cortex, RMC red nucleus magnocellular part, RPC red nucleus parvicellular part, Rt reticular thalamic nucleus, RSA retrosplenial agranular cortex, RSG retrosplenial granular cortex, Re reuniens thalamic nucleus, M2 secondary motor cortex, S2 secondary somatosensory cortex, Shi septohippocampal nucleus, S subiculum, SI substantia innominata, SNC substantia nigra compact part, SNR substantia nigra reticular part, SCh suprachiasmatic nucleus, SO supraoptic nucleus, VO ventral orbital cortex, VP ventral pallidum, VPM ventral posteromedial thalamic nucleus, VTA ventral tegmental area, VL ventrolateral thalamic nucleus, VMH ventromedial hypothalamic nucleus, VM ventromedial thalamic nucleus, ZI zona incerta.

Figure 4. Reduction in functional connectivity in the extended amygdala and central stress response circuits in the GRi mouse model as compared to the wild-type mice. Based on the multidimensional scaling analysis, brain regions in these circuits were examined pair-wise for correlation in oxidative metabolism. Numbers are Pearson correlation coefficients. 

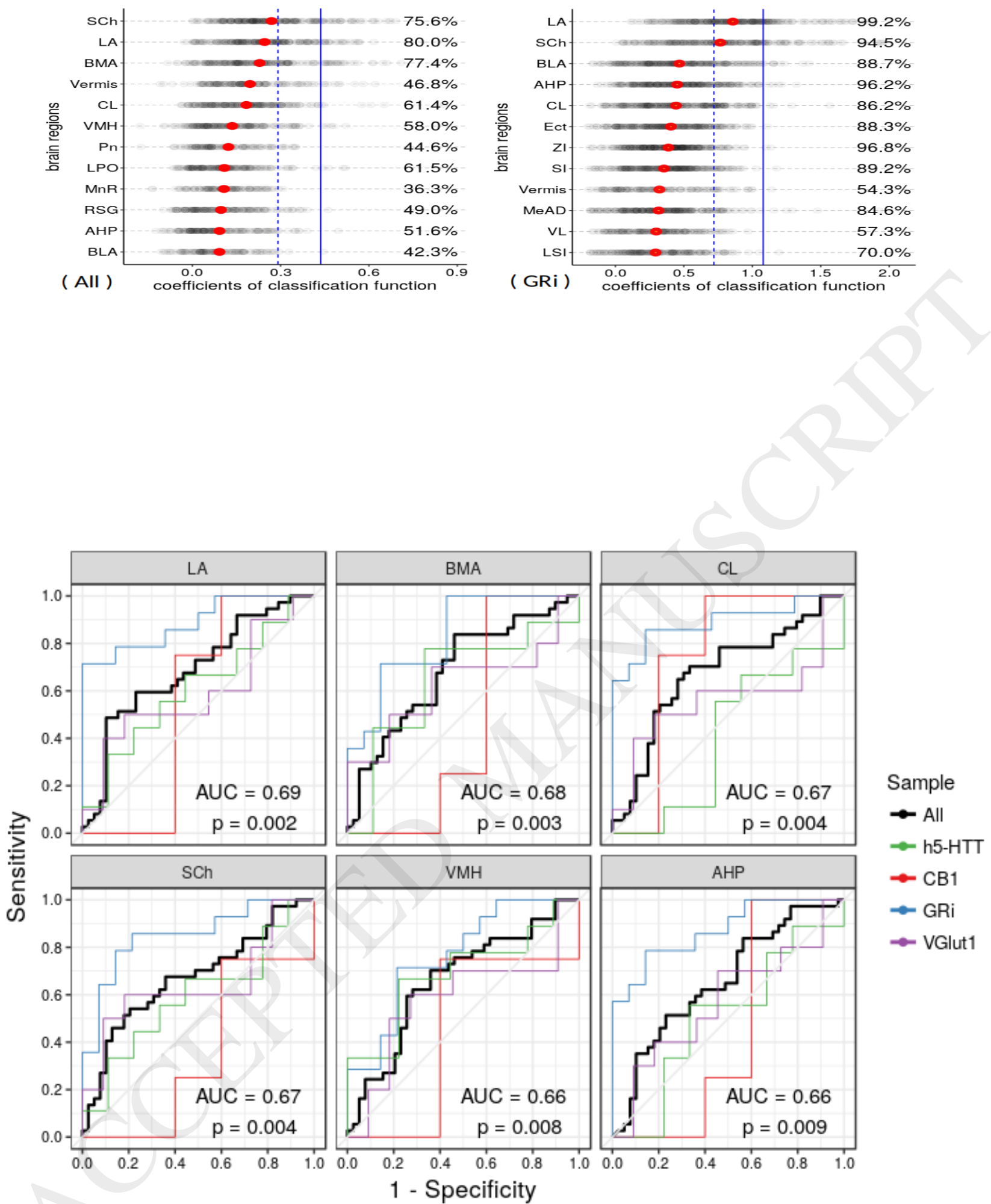


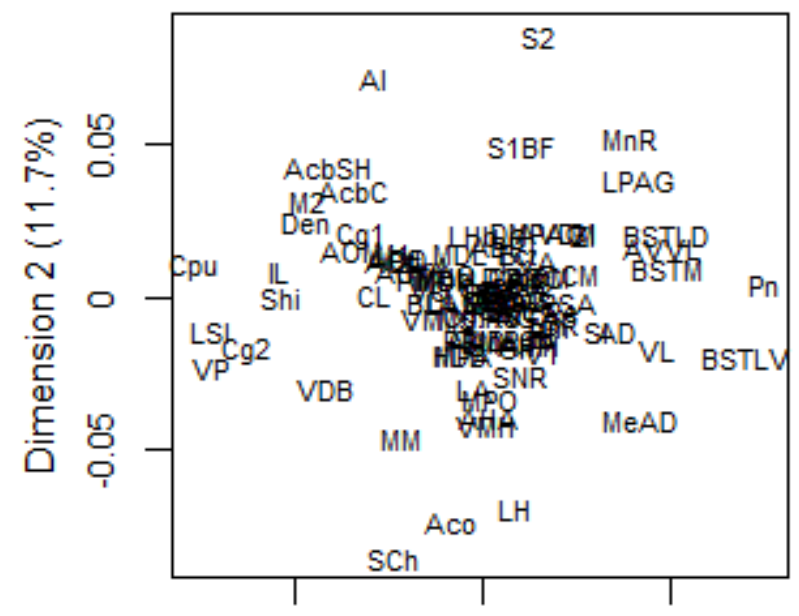
( All )
$-0.05$
0
0.05
Dimension 1 (14.7\%)

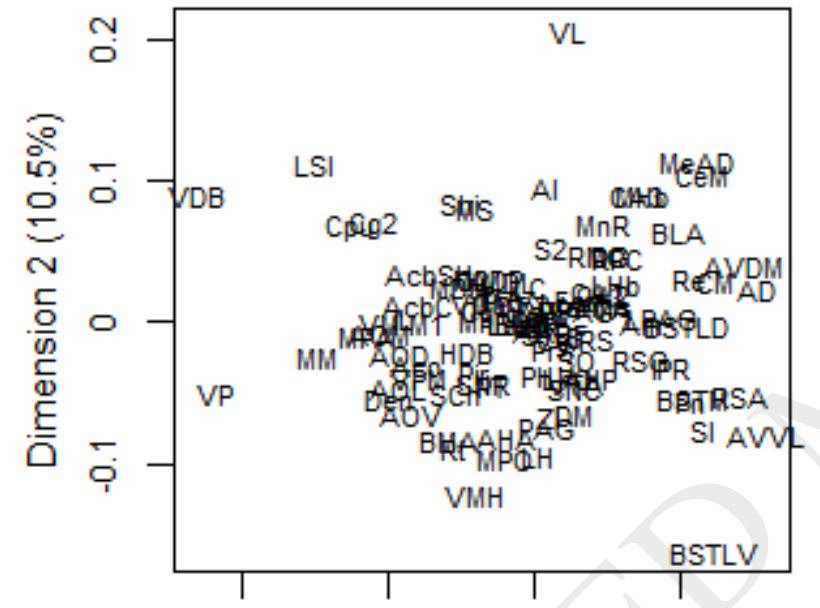

( GRi ) $\begin{array}{cccc}-0.2 & -0.1 & 0 & 0.1 \\ \text { Dimension 1 (19.6\%) }\end{array}$ 


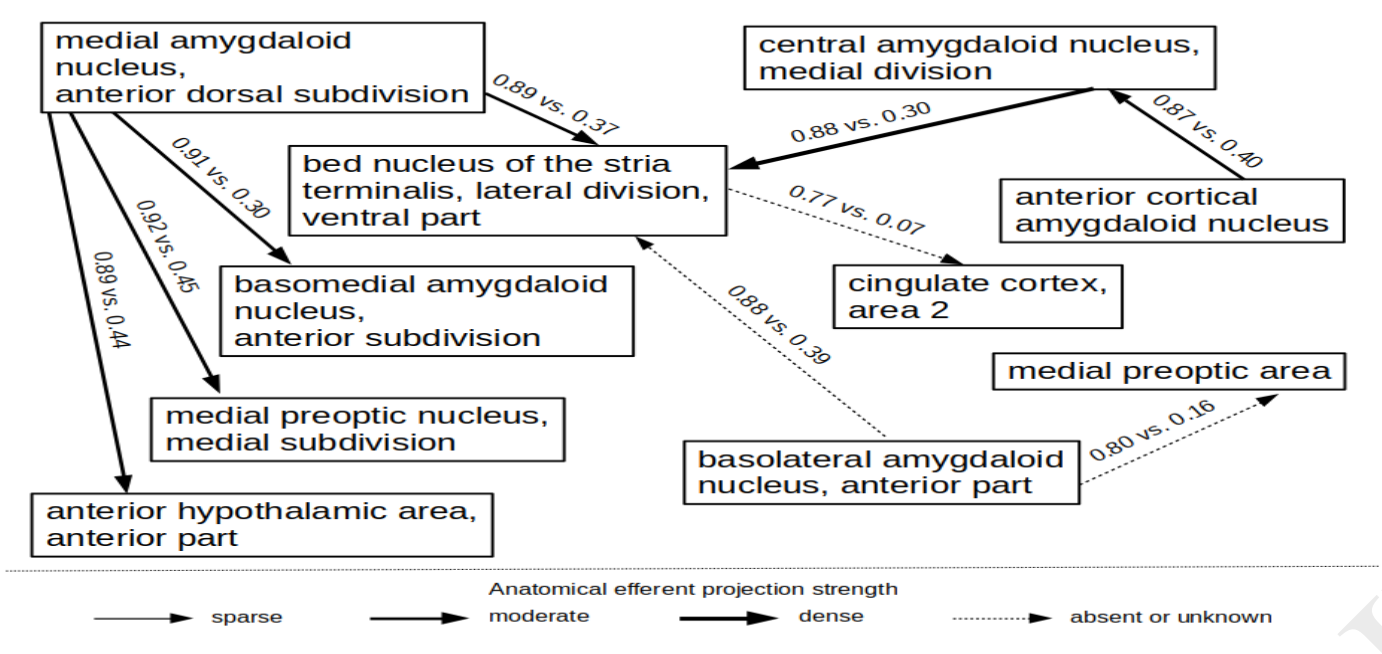

